The book is in the first place written for scientific workers who wish to engage in research on the structure of molecules. There is therefore no attempt to derive the theoretical formulæ from first principles, and at every step the theory is put to the test by comparison with experiment. The virtues of the different methods, the errors to be avoided and the corrections to be applied are very thoroughly discussed; but perhaps a little too much space is taken up by the detailed description of the author's own work as compared with the work of others. Those readers who wish to use the book as a source of general information on the subject would have preferred to find a short systematic theoretical treatment of the scattering of $\mathrm{X}$-rays and electrons on the basis of quantum mechanics, which would greatly help towards a better understanding of the connexions between the different forms of the scattering phenomenon.

There are a few minor errors; for example, the statement on p. 21 that the frequencies in the spectrum of the incoherently scattered radiation are all smaller than the primary, or the statement on p. 26 that in coherent scattering the primary photon is first captured by the atom and then re-emitted again in its entirety. The discussions on pp. 12 and 28 on the quantum mechanical wave function are incorrect as they omit to take into account that these functions are complex and not real. The explanation of the resonance effect produced by the $K$-frequencies on the dispersion of the atomic scattering factor on p. 38 is also misleading.

Apart from these minor defects the book is very lucidly written and accompanied by a great number of clear diagrams and illustrations. It can be warmly recommended.

R. FURTH

\section{CHEMISTRY AND LIGHT}

\section{The Chemical Aspects of Light}

By E. J. Bowen. Second edition revised. Pp. iv + 300. (Oxford: Clarendon Press; London: Oxford University Press, 1946.) 15s. net.

NR. BOWEN writes in his preface that "the 1 second edition of this book has the same aim as the first : to present modern ideas of matter and light as far as possible in non-mathematical form. -.. Wave mechanics is a difficult subject which cannot by its intrinsic nature be conveyed in a pictorial form, yet such a theoretically unsound task is what is attempted here". There can be no doubt that Mr. Bowen has succeeded in his task; moreover, the task itself has increased in magnitude since the first edition was published; and, in general, wave mechanical topics have been treated more deeply in the second edition.

The arrangement of the book remains approximately the same; but the text has been largely rewritten and extended. The early chapters on light and waves, and light and light sources remain sensibly unchanged. Chapter 3 on the absorption and emission of light has been considerably enlarged. The main addition is a discussion of molecular orbitals, and their construction from and relation to the corresponding atomic orbitals. In the course of this, carbon-carbon single and multiple bonds, and conjugated systems, including benzene, are dealt with. There follows a very interesting account of 'colour', in which the position, shape and intensity of absorption bands are related to molecular structure, molecular orbital and resonance methods being used. Chapter 4, on the transformation of absorbed radiation, now includes a discussion of the kinetics of the quenching of fluorescence in solution, and the information which may be obtained by a study of quenching about reactions in solution with high velocity constants.

Typical additions in the remaining chapters are the discussion of a number of photochlorinations, and geometrical isomerizations (Chapter 6) and the recent Russian work on photosynthesis (Chapter 7). There are also two additional appendixes: one on filter solutions, and a comprehensive list of "Experiments with Light of Chemical Interest".

For readers who are not familiar with the first edition, it may be stated that the book is written in an extremely lucid and attractive style, and will be useful not only to students as an introduction to several aspects of chemical physics, but also to practical photochemists. The author is to be congratulated on adding substantially to the value of an already excellent book. It is a pity that references to original papers have been omitted from the second edition.

The price is very reasonable.

C. H. BAMForD

\section{LEGENDRE POLYNOMIALS}

\section{British Association for the Advancement of} Science

Mathematical Tables. Part-Volume A: Legendre Polynomials. Prepared by the Committee for the Calculation of Mathematical Tables. Pp. A42. (Cambridge : At the University Press, 1946.) 8s. 6d. net.

THIS is the first of a post-war series of mathematical tables published for the British Association, in response to the ever-growing demand arising from research work in mathematics and physics.

It is designated Part-Volume $\mathbf{A}$, and appears in conjunction with a companion volume on the Airy integral. Unlike the other recent publications dealing with the tabulation of Legendre functions, this book makes no reference to the associated functions and is concerned only with the polynomial solution of Legendre's differential equation :

$$
\left(1-x^{2}\right) \frac{d^{2} y}{d x^{2}}-2 x \frac{d y}{d x}+n(n+1) y=0,
$$

for integers $n$ ranging from 2 to 12 inclusive.

In the first section, tables of $P_{n}(x)$ for $n=2$ to 6 are provided for the range $x=0$ to 6 at intervals of 0.01 . The entries are, in general, accurate to seven significant figures, and second differences are supplied.

The second section deals with the next three functions with similar accuracy, fourth differences being included where necessary. The remaining three functions are tabulated in the final section.

The whole production has been supervised by Dr. L. J. Comrie and is prefaced by his introduction, which describes the material on which the tables are based, together with the methods used in their construction. With his characteristic thoroughness, Dr. Comrie has provided means for ensuring the complete accuracy and legibility of every figure, and one can look forward with confidence and pleasure to future publications of a similar nature. J. H. PEARCE 